\title{
Asymmetric central processing of sensory stimuli in idiopathic focal dystonia
}

\author{
R.A. Grünewald, J. Shipman, Y. Yoneda and H.J. Sagar \\ Department of Clinical Neurology, University of Sheffield, Royal Hallamshire \\ Hospital, Sheffield, UK \\ Correspondence to: R.A. Grünewald, Department of Clinical Neurology, \\ University of Sheffield, Royal Hallamshire Hospital, Glossop Road, Sheffield, \\ S10 2JF, UK
}

\begin{abstract}
Idiopathic torsion dystonia is characterized by persistent abnormalities of posture. We tested the hypothesis that abnormal sensorimotor processing is involved pathophysiologically by looking for asymmetry of sensory processing in patients with asymmetric symptoms. Sixteen patients with torticollis (ten with head turning to the right and six to the left), seven with simple writer's cramp and 19 healthy control subjects were tested. The tasks involved matching one of five lengths of stick or weights presented to one hand with sticks and weights chosen by the other hand. All experiments were performed with the subject blindfolded. Patients with torticollis tended to underestimate weights presented to the hand away from which the head tended to turn. Control subjects showed no significant tendency to overestimate or underestimate lengths or weights with either hand, and dystonic patients showed no tendency to overestimate or underestimate lengths. Those with writer's cramp underestimated weights when the stimulus was presented to the affected hand. An asymmetry can thus be detected in muscles remote from the site of dystonia, indicating a generalized abnormality of sensorimotor processing.
\end{abstract}

Keywords: Dystonia - Psychomotor performance

\section{INTRODUCTION}

Idiopathic focal dystonia is a disorder of uncertain aetiology which is characterized by sustained, involuntary muscle contraction. There is an increasing body of evidence that idiopathic dystonia involves a disorder of sensation. For example, a characteristic clinical feature is the geste antagonistique, where increased somatosensory feedback, in the form of a finger placed lightly on the chin, may partially correct the dystonia in patients with torticollis. Sensory stimuli, especially painful trauma involving muscle or tendon strain rather than sharp injury, may precede the onset of dystonia (Poersch and Schlegel, 1996). Abnormalities of somatosensoryevoked potentials (Reilly et al., 1992) and regional cerebral blood flow changes in response to vibration (Tempel and Perlmutter, 1990, 1993) have been reported in dystonia. Thus, abnormal sensory function is implicated in both the aetiology and pathophysiology of idiopathic dystonia.

If dystonia is predominantly a sensory disorder, the presence of focal, asymmetric symptoms would imply an asymmetry of sensory processing. If this were the case, patients might show an abnormal asymmetry in matching sensory inputs from left and right sides which would vary according to which side of the body was predominantly affected. This hypothesis was tested using a series of simple experiments involving matching of weights and lengths which were estimated with each hand separately and without visual input.

\section{METHODS}

\section{Subjects}

The study involved 23 patients with idiopathic dystonia (12 male, 11 female, median age 55.7 years, range 36.9-78 years) and 19 healthy control subjects (seven male, 12 female, median age 50.0 years, range 29.0-68.1 years); the patients were recruited from the movement disorders outpatient clinic at the Royal Hallamshire Hospital, Sheffield, UK. Control subjects comprised spouses of dystonic subjects, staff and students at the Royal Hallamshire Hospital. None had evidence of other neurological disease or a family history of dystonia, nor were they taking psychoactive medication at the time of the study. Sixteen patients had torticollis, (6 head turning to the left and 10 to the right) and seven patients had simple writer's cramp. 
The median duration of symptoms was five years (range three months to 31 years). All those with torticollis had received local injections of botulinum toxin to the overactive muscle groups in the neck within the last three months. None of the patients with simple writer's cramp had ever received treatment with botulinum toxin. Subjects were asked which hand they habitually used for writing before the onset of dystonia. Two of the subjects with writer's cramp, one with torticollis and two of the healthy control subjects were lefthanded and the remainder were right-handed. The left hand was dystonic in the left-handed patients with writer's cramp. Informed consent was obtained from all subjects, and the research protocol had been approved by the local ethics committee.

\section{Tasks}

The tasks involved matching one of five weights or lengths of orange stick presented to one hand with weights or sticks chosen by the other hand. The underestimate or overestimate of each weight or length was recorded. Subjects were blindfolded and seated comfortably with elbows resting on the table. In the first experiment, they were instructed that one of five plastic bottles of identical appearance, but slightly different weights, would be placed in the palm of one upturned hand. Two sets of five closely graded weights were made for this experiment by using different quantities of plaster of paris in identical plastic sample tubes that were $5.6 \mathrm{~cm}$ in length and $1.5 \mathrm{~cm}$ in diameter. The plaster of paris was solidified by the addition of a small amount of water to prevent movement of the contents acting as a perceptive clue. Bottles had screw tops which gave a tight seal to avoid changes in weight due to evaporation; they were checked before and after the experiments.

Trials were performed on three healthy volunteers from the University of Sheffield to find a suitable weight difference. The minimum weight difference which produced $90 \%$ correct matching in these volunteers was $2.7 \mathrm{~g}$. For convenience, a weight range of $4.9 \mathrm{~g}$ to $15.7 \mathrm{~g}$ was selected.

Subjects were asked to choose a bottle with the other hand, from five in front of them, which most closely matched the stimulus bottle in weight. They were asked to feel through the full set of weights, in ascending weight order, in front of them before deciding on the best match. The subjects were told that they could handle the presentation stimulus bottle with only one hand, and that they could feel the matching bottles with the other hand only. They were allowed to move either bottle around in the respective hand freely and to move their hands in space as long as the hands or weights did not touch. Subjects were asked to assess the chosen weight against the presented weight before making a final decision by holding it in the upturned palm with the elbow on the table so that both weights were being assessed in the same manner. The order of presentation of the five stimulus bottles was chosen at random. Each stimulus was presented once to each hand. After subjects had picked the weights they believed to match each of the five randomly-presented weights, the test was repeated by presenting weights to the opposite hand. No time limit was imposed, but subjects were asked to make an instinctive decision if they. were having problems in picking a weight. Subjects were not given any feedback on their performance during the test.

The second task was analogous but involved matching lengths of orange stick. Two identical sets of five closely-graded lengths of orange stick were made for this test. Wooden orange sticks were used as they were readily available and light enough to avoid differentiation of lengths on the basis of weight. A length difference of $5 \mathrm{~mm}$ and a range of $51 \mathrm{~mm}$ to $71 \mathrm{~mm}$ was used, producing a range in weight of $0.9 \mathrm{~g}$ to $1.79 \mathrm{~g}$, which was below the limits of discrimination of difference on the basis of weight alone. The lengths were chosen so as to be short enough to be assessed between thumb and forefinger of one hand. Subjects were blindfolded and presented with one of five lengths of orange stick, chosen at random, in one hand. They were required to choose the length that they believed matched that of the first stick in the other hand from a set of five in ascending order of length which were placed on the table in front of them.

In the standard testing protocol, subjects adopted the head position they found most comfortable. In a sub-group of six healthy control subjects only, the length- and weight-matching tasks were repeated after the subjects had been asked to maintain the head turned to the right or the left. All subjects were tested in the same room using the same equipment and sitting on the same chair at the same table. The room was otherwise unoccupied to avoid extraneous auditory stimulation.

\section{RESULTS}

The mean underestimate or overestimate of lengths and weights in the right hand relative to the left for each subject was compared between the three subject groups (torticollis to the right, torticollis to the left and healthy control subjects) and between the affected (i.e. dominant) and unaffected hand in patients with writer's cramp using analysis of variance with Fisher's exact test. 
TABLE I. Matching of weights and lengths

\begin{tabular}{lllll}
\hline & $\begin{array}{l}\text { Torticollis } \\
\text { to the right } \\
\mathrm{n}=10\end{array}$ & $\begin{array}{l}\text { Torticollis } \\
\text { to the left } \\
\mathrm{n}=6\end{array}$ & $\begin{array}{l}\text { Writer's } \\
\text { cramp } \\
\mathrm{n}=7\end{array}$ & $\begin{array}{l}\text { Healthy control } \\
\text { subjects } \\
\mathrm{n}=19\end{array}$ \\
\hline $\begin{array}{l}\text { Mean underestimate of weight with right } \\
\text { hand }(\mathrm{g} \pm \text { standard error of mean) }\end{array}$ & $-1.03 \pm 0.34 * * *$ & $0.72 \pm 0.74^{\star *}$ & $0.50 \pm 0.42^{*}$ & $0.38 \pm 0.18$ \\
$\begin{array}{c}\text { Mean underestimate of length with right } \\
\text { hand (mm } \pm \text { standard error of mean) }\end{array}$ & $-0.4 \pm 0.80 \mathrm{~ns}$ & $0.92 \pm 1.16 \mathrm{~ns}$ & $-0.21 \pm 0.82 \mathrm{~ns}$ & $0.79 \pm 0.44$ \\
\hline
\end{tabular}

${ }^{\star \star *} p<0.002$ with respect to control subjects, ${ }^{\star *} p<0.004$ with respect to torticollis to the right, ${ }^{*} p<0.05$ with respect to the left, ns $=$ not significant by ANOVA with Fisher's PLSD.



FIG. 1. Underestimate of lengths of right/dominant hand.

Results were expressed as mean underestimates with the dominant/dystonic hand. This is the difference between the value of weight or length chosen by left/non-dominant hand and the value of weight or length chosen by right/dominant hand. The results are shown in Table I. Control subjects showed no significant tendency to overestimate or underestimate lengths or weights. There was no significant tendency to overestimate or underestimate the lengths of orange stick in dystonics (Fig. 1). Patients with torticollis underestimated weights presented to the hand away from which the head turned (torticollis to the left $0.72 \mathrm{~g} \pm 0.74 \mathrm{~g}$, torticollis to the right,
$-1.03 \mathrm{~g} \pm 0.34 \mathrm{~g}$ ), and those with writer's cramp underestimated weights by $0.50 \pm 0.42 \mathrm{~g}$ when the stimulus was presented to the affected hand (Fig. 2). In six control subjects, head position (rotated to right or left) per se did not significantly affect the matching of lengths or weights. The results are shown in Table II.

No specific observation protocol was adopted for analysis of the strategy employed by subjects undertaking the tasks. However, observation of the subjects during the experiment showed that, during the lengthmatching task, subjects would usually compare the lengths of sticks held between thumb and forefinger, sometimes held in the hand and sometimes with the 


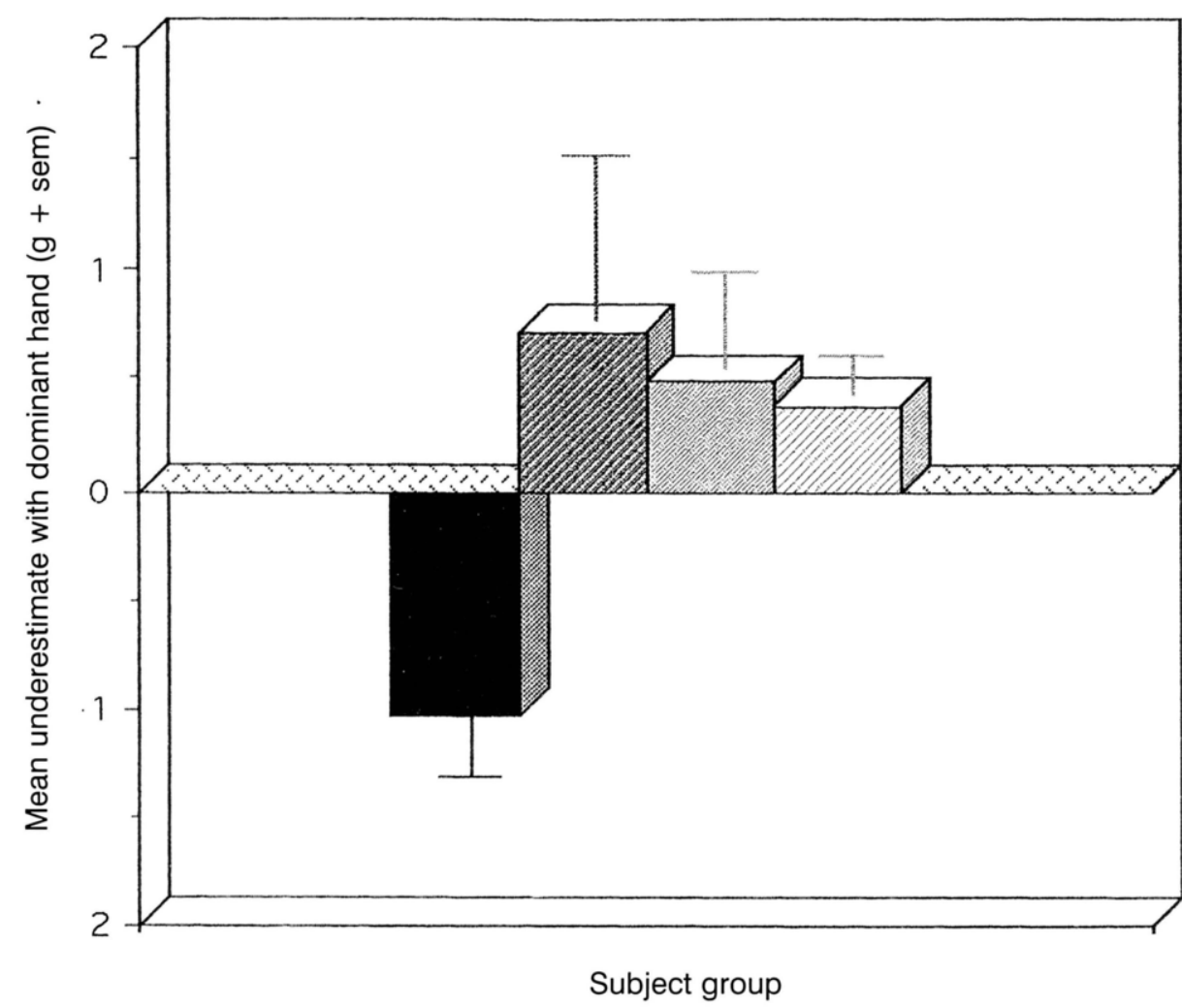

- torticollis to right

torticollis to left

Writer's cramp

Controls

FIG. 2. Underestimate of weight with right/dominant hand.

TABLE II. Effect of head rotation in healthy control subjects on ability to match lengths and weights

\begin{tabular}{llll}
\hline & $\begin{array}{l}\text { head straight } \\
\mathrm{n}=6\end{array}$ & $\begin{array}{l}\text { head rotated to right } \\
\mathrm{n}=6\end{array}$ & $\begin{array}{l}\text { head rotated to left } \\
\mathrm{n}=6\end{array}$ \\
\hline $\begin{array}{l}\text { Mean underestimate of weight with right hand } \\
\text { (g } \pm \text { standard error of mean) }\end{array}$ & $1.3 \pm 0.97$ & $1.25 \pm 0.80 \mathrm{~ns}$ & $1.17 \pm 0.44 \mathrm{~ns}$ \\
$\begin{array}{l}\text { Mean underestimate of length with right hand } \\
\text { (mm } \pm \text { standard error of mean) }\end{array}$ & $0.4 \pm 0.48$ & $0.13 \pm 0.27 \mathrm{~ns}$ & $0.59 \pm 0.44 \mathrm{~ns}$ \\
\hline
\end{tabular}

$\mathrm{ns}=$ not significant: underestimates do not differ with head position by ANOVA.

sticks resting on the table surface. In contrast, the weight-matching task often involved estimation of inertia during movement of the weight up and down against gravity.

\section{DISCUSSION}

Idiopathic focal dystonia is a disorder characterized by more or less sustained abnormalities of posture, most commonly involving the turning of the head to one side (torticollis). Its aetiology remains obscure and, for many years, it was considered to be 'hysterical' in origin. More recently, neurophysiological abnormalities have been demonstrated in dystonic patients, establishing that it has an 'organic' basis (Nakashima et al.,
1989). It is not caused simply by spasm or overactivity of a muscle or groups of muscles. Patients treated with botulinum toxin, which weakens the overactive muscles, may continue to experience abnormal head posture even when the muscles responsible for head rotation are severely weakened in comparison with their antagonists. On the other hand, improvement of symptoms in idiopathic focal dystonia usually occurs when the affected part of the body is touched (geste antagonistique), for example, torticollis improves if the chin is lightly touched by the patient's hand. This implies that the symptoms are at least partially due to a sensory disorder. Therefore, muscular weakness induced by botulinum toxin may not alter abnormal posture, although it would improve associated muscle 
pain caused by inappropriate co-activation of agonist and antagonist muscles, but additional postural sensory feedback provided by touching the chin will alter abnormal posture. The abnormal posture is postulated to be the result of abnormal sensorimotor integration; for example, misinterpretation or imbalance of postural information from the muscles of the neck.

These experiments showed that patients with focal dystonia affecting the neck or hand showed an asymmetry of sensorimotor processing. This abnormal asymmetry can be detected in the limbs remote from the site of dystonic symptoms and it is, therefore, unlikely to be attributable to postural abnormalities. This is confirmed by the experiments on healthy control subjects which showed that adopting a posture with the head turned to the left or right did not significantly affect the ability to match lengths or weights. These results imply that the demonstrated abnormality of sensorimotor processing may occur independently of dystonic symptoms, i.e. that it may represent a preexisting abnormality rather than an effect of the dystonia or a direct cause of the symptoms.

Patients with writer's cramp and torticollis to the left underestimate weights presented to the right hand. If it can be assumed that the affected arm in patients with writer's cramp undergoes more abnormal sensory processing, it follows that sensory information from the side of the body away from which the head turns in torticollis undergoes more abnormal processing.

Treatment with botulinum toxin may have complex effects in dystonia and the treatment may act predominantly on the intrafusal or extrafusal muscle fibres. Patients sometimes report alteration of symptoms quite remote from the site of injection of botulinum toxin. Although the significance of such changes is difficult to interpret, systemic action of the drug is a possibility, especially as the doses required to influence the intrafusal muscle fibres of muscle spindles are probably very small. It is unlikely that the results of this study are due to prior treatment of the patients with botulinum toxin for two reasons. Firstly, injections of botulinum toxin in the patients with torticollis were given bilaterally into the sternomastoid and contralateral posterior cervical muscles which are some distance from the hands undertaking the tasks. Although local botulinum toxin injections may induce systemic effects, these are unlikely to be consistently lateralized. Secondly, and more importantly, patients with writer's cramp who had not received botulinum toxin also showed abnormalities of weight matching.

There have been reports of abnormal somatosensory responses in idiopathic focal dystonia (Tempel and Perlmutter, 1990, 1993; Reilly et al., 1992, especially to vibratory stimuli which preferentially stimulate muscle spindle Ia afferents, (Goodwin et al., 1972; Tempel and Perlmutter, 1990, 1993). Vibratory stimuli have been shown to induce dystonic symptoms in patients with writer's cramp (Tempel and Perlmutter, 1993; Kaji et al., 1995). In certain circumstances, vibratory stimuli may relieve dystonic symptoms in this group of patients (Leis et al., 1992). Such symptoms may also be relieved by intramuscular injections of lignocaine, which block $\gamma$ motor neurone and Ia afferent activity (Kaji et al., 1995). Electrophysiological studies of Ia afferent activity have demonstrated abnormally reduced reciprocal inhibition in dystonic subjects, suggesting reduced Ia presynaptic inhibition (Nakashima et al., 1989), and this may also be found in regions remote from the symptomatic area in dystonic patients. These observations are, therefore, consistent with a role of muscle spindle Ia afferents in the pathophysiology of dystonia. Ia afferent discharges are probably responsible for the sensation of movement which is produced by vibration of a muscle tendon or belly (Matthews, 1977) and, thus, abnormal sensation of joint movement may be a feature of dystonia.

Our results confirm that abnormalities of sensation can be detected in dystonics and that these vary according to the lateralization of the abnormal posture. It is apparent that the sensory abnormalities detected were not identical for all sensory modalities. Matching of lengths was not significantly impaired in the dystonic subjects, in contrast to matching of weights. During the length-matching task, subjects often compared the lengths of sticks held between thumb and forefinger or lying on the table surface. In contrast, the weight-matching task involved estimation of inertia during movement of the weight. The former task was, therefore, greatly dependent on static position sense whereas the latter involved more movement of the hands. The impairment of weight matching, but not length matching, may be considered to be an abnormal perception of movement rather than of position. This is consistent with dystonia being an abnormality of movement control rather than of static posture. These results are consistent with a role of abnormal Ia afferent processing in dystonia (Nakashima et al., 1989). The focal nature of dystonia may be explained by an abnormal asymmetry of muscle spindle afferent processing.

\section{REFERENCES}

Goodwin GM, McCloskey DI and Matthews PBC (1972). The contribution of muscle afferents to kinaesthesia shown by vibration induced illusions of movement and by 
the effects of paralysing joint afferents. Brain 95, 705-748.

Kaji R, Rothwell JC, Katayama M, Ikeda T, Kubori T, Kohara N, Mezaki T, Shibasaki H and Kimura J (1995). Tonic vibration reflex and muscle afferent block in writer's cramp. Annals of Neurology 38, 155-162.

Leis AA, Dimitrijeciv MR, Delapasse JS, Sharkey PC (1992). Modification of cervical dystonia by selective sensory stimulation. Journal of the Neurological Sciences 110, 79-89.

Matthews PBC. (1977) Muscle afferents and kinaesthesia. British Medical Bulletin, 137-142.

Nakashima K, Rothwell JC, Day BL, Thompson PD, Shannon K and Marsden CD (1989). Reciprocal inhibition between forearm muscles in patients with writer's cramp and other occupational cramps, symptomatic hemidystonia and hemiparesis due to stroke. Brain 112, 681-697.
Poersch M, Schlegel U (1996). Post-traumatic dystonia of peripheral origin: a meta-analysis of 68 case reports. Movement Disorders 11 (Suppl 1), 207.

Reilly JA, Hallett M, Cohen LC et al. (1992). The N30 component of somatosensory evoked potentials in patients with dystonia. Electroencephalograph and Clinical Neurophysiology 84, 243-247.

Tempel LW, Perlmutter JS (1990). Abnormal vibrationinduced cerebral blood flow responses in idiopathic dystonia. Brain 113, 691-707.

Tempel LW, Perlmutter JS (1993). Abnormal cortical responses in patients with writer's cramp. Neurology 43, 2252-2257.

(Received 10 October 1996; accepted as revised 27 November 1996) 


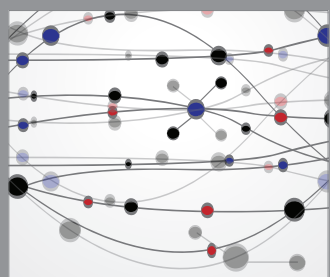

The Scientific World Journal
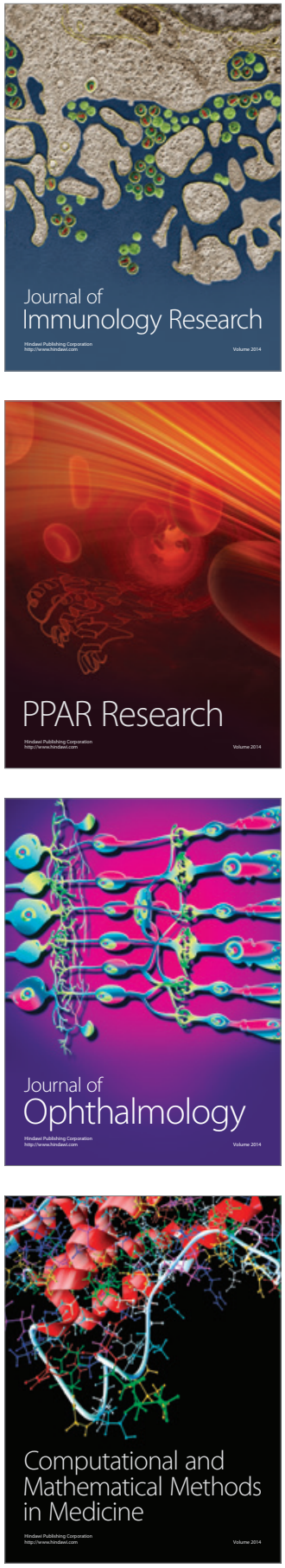



Gastroenterology

Research and Practice
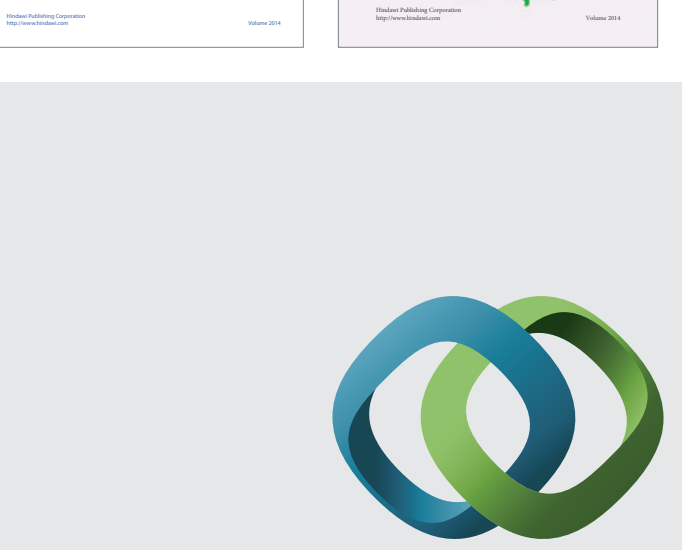

\section{Hindawi}

Submit your manuscripts at

http://www.hindawi.com


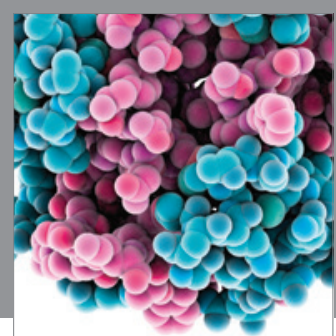

Journal of
Diabetes Research



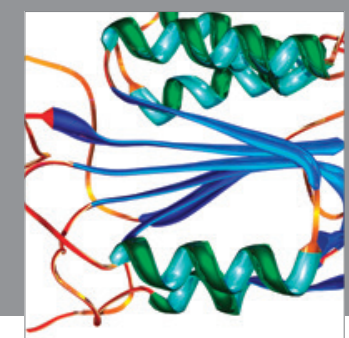

Disease Markers
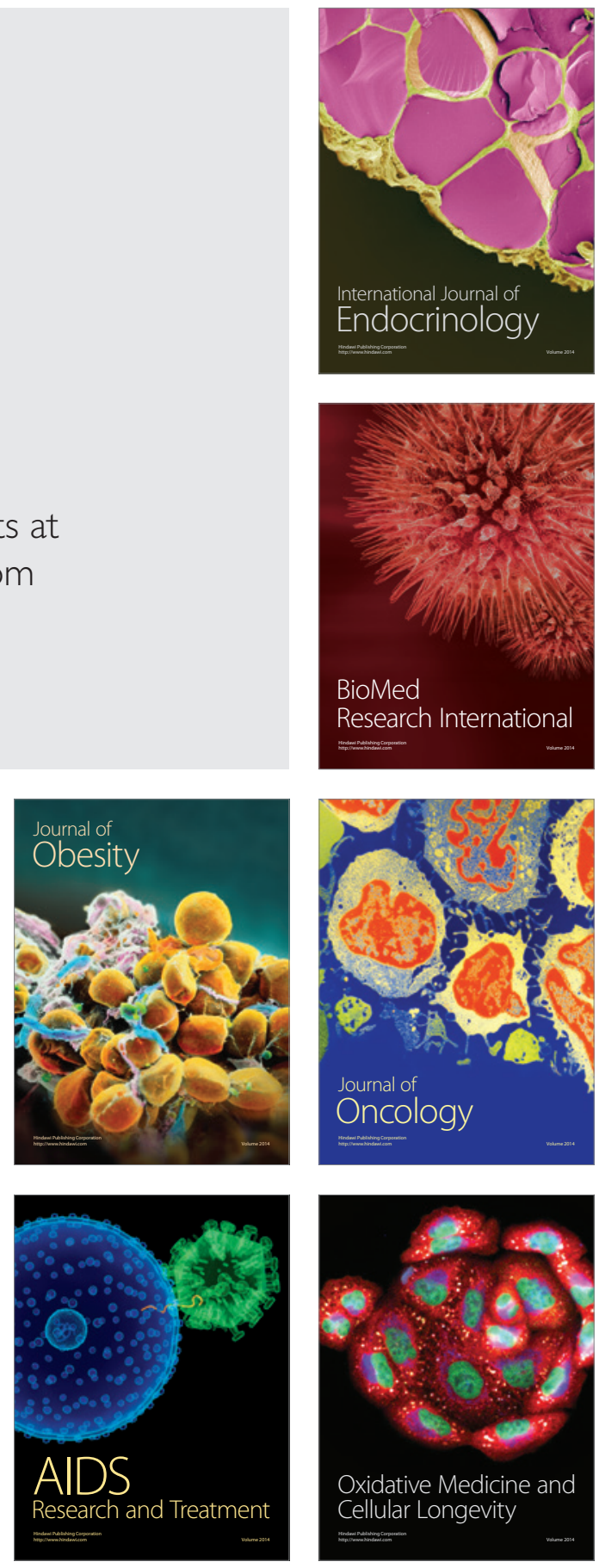